\title{
Evaluating Enterprise Risk in a Complex Environment
}

\author{
Ivan De Noni ${ }^{1}$, Luigi Orsi ${ }^{2}$, Luciano Pilotti ${ }^{1}$ \\ ${ }^{1}$ University of Milan, DEAS, Milan, Italy; ${ }^{2}$ University of Padua, Padua, Italy. \\ Email: ivan.denoni@unimi.it
}

Received June $11^{\text {th }}, 2010$; revised July $19^{\text {th }}, 2010$; accepted August $21^{\text {st }}, 2010$.

\begin{abstract}
This paper examines the relationship between operational risk management and knowledge learning process, with an emphasis on establishing the importance of statistical and mathematical approach on organizational capability to forecast, mitigate and control uncertain and vulnerable situations. Knowledge accumulation reduces critical situations unpredictability and improves organizational capability to face uncertain and potentially harmful events. We retain mathematical and statistical knowledge is organizational key factor in risk measuring and management process. Statistical creativity contributes to make quicker the innovation process of organization improves exploration capacity to forecast critical events and increases problem solving capacity, adaptation ability and learning process of organization. We show some important features of statistical approach. First, it makes clear strategic importance of risk culture within every level of organization; quantitative analysis support the emergence of latent troubles and make evident vulnerability of organization. Second, innovative tools allow to improve risk management and organizational capability to measure total risk exposition and to define a more adequate forecasting and corrective strategy. Finally, it's not so easy to distinguish between measurable risk and unmeasurable uncertainty, it depends on quantity and quality of available knowledge. Difficulty predictable extreme events can bring out crisis and vulnerable situations. Every innovative approach which increases knowledge accumulation and improves forecasting process should be considered.
\end{abstract}

Keywords: Complexity, Extreme Events, Operational Losses, Quantitative Management

\section{Introduction}

In literature we find out paper explaining mathematical techniques application to operational risk evaluation and other concerning risk management principles and features. What we would like to do in this paper is to merge this too often separated concepts. Thus, in the first part, we describe some aspects of operational risk mainly with respect to relationship between uncertainty and corporate learning process; in the second one, we argue general mathematical approaches implications on operational risk and knowledge management and then we show an innovative mathematical method and exploit its advantages, disadvantages and further extensions.

In modern economic context, knowledge management is a more and more important resource for success of firms. However, getting information is only the first step for a long time sustainable development. In a dynamic system in fact, knowledge may result just a short time vantage because its non-excludability property makes its transfer and competitors' imitation easy [1]. To acquire competitive vantages, firms have to develop and continually improve suitable capabilities and organize routines to control, manage and use the knowledge in a profitable way [2].

Thus, knowledge management becomes progressively the success key for securing organization's sustainability [3], even if a successful learning process needs a good adaptation capability at the same time. Only a supple firm is able to adapt its cognitive patterns to environment and market changes [4] quickly and properly enough to develop an effective generative learning process for new knowledge creation [5]. Knowledge management is a dynamic capability [6]; a successful strategy in the short term may become less efficient in the long one if the firm is unable to increase continually its knowledge, improving competences and innovating competitive advantage.

So we can conclude efficient knowledge management and adaptation capability are both required to organiza- 
tion sustainability. When these conditions are both satisfied, an organization can implement a generative learning process to preserve new knowledge creation and innovation. In this perspective, statistical analysis and mathematical models are some of possible tools which can help firms to improve knowledge accumulation and decision making processes. More and more detailed and reliable forecasting models, in fact, permit to better predict environment changes and manage correlated risk, reducing uncertainty and increasing the organization's problemssolving capability.

\section{Organization, Risk and Knowledge}

Each enterprise can be thought as a socio-economic organization, headed by one or more persons with a propensity to risk $[7,8]$. So risk is an integral part of the firm. The increasing complexity of modern society makes risk a particularly critical factor because company management is often unable to face it [9-11]. When we talk about risk we refer in particular to operational risk $^{1}$.

If you consider the more and more critical role of risk in company governance, it's easy to understand because overlooking or thinking risks in simplistic way may lead to inadequate exposition or unconscious acceptance by the organization. In contrast, when risk is implemented in the corporate culture, it develops into a production factor and its suitable management becomes an essential part of value creation chain [12]. Just when the concept of risk is integrated in corporate culture, you can identify the basis for prudent and responsible corporate governance [13].

However we need to distinguish between governance and management of business risk. They are two interconnected but different moments of decision-making process. Government takes care of placing the organizational bounds qualifying the logic of value creation and the maximum tolerable risk, while management tends to decompose the overall business risk in a variety of risks, following the stages of assessment, treatment and reassessment of all relevant risk [14].

In other words, risk governance relates to risk culture sharing throughout the all organization as well as to reduce risk overlooking. Risk management relates to every process or technique which allows to mitigate or remove risk for organization. In first instance potential efficient management depend on efficient governance.

Thus, a careful administration of risks leads to some preliminary considerations:

\footnotetext{
${ }^{1}$ According to Basel Committee [15] operational risk is defined as the risk of loss resulting from inadequate or failed internal processes, people and systems or from external events.
}

- A corporate governance is based on a complex and dynamic mix of risks linked inextricably to the enterprise system;

- Usually a business risk cannot be fully cancelled;

- Each action to reduce risk exposition involves an organizational cost and brings out other hazards;

- The sustainability of any risk depends on the amount of enterprise knowledge and competences.

Last point focuses on the relationship between risk and knowledge. The risk reflects the limitations of human knowledge or bias, indicating the possible events to which it is exposed due to the combination of their choices, external conditions and the flow of time. Just if knowledge was complete and perfect, firm would operate in conditions of certainty [16]. So risk and knowledge are each other mutually dependent: the risk marks the limit of knowledge and it allows the perception of risk [17]. Over time, the learning process of an organization leads to a better understanding of reality and a more awareness of risk and thus allows to reduce uncertainty and to develop greater risk management and forecasting capabilities.

In literature we found three different approaches which try to define the relationship between knowledge and risk: scientist current, social current and critical current.

The first one is based on logical-mathematical approaches and believes in primacy of knowledge on risk $[18,19]$. The second one retains limits of knowledge and social interaction processes make the risk a feature of contemporary society, where knowledge contribution merely asserts organizational inability to eliminate risk [20]. The critical current based its thesis on knowledge and risk dynamics, where risk cannot be eliminated but it can be reduced or contained [12]; so the learning creative process of a business organization needs to track the business risk, trying to transform or mitigate it through company skills and competences, which organizational knowledge renews over time.

Spread risk culture to any firm level means developing an attitude to adaptation and risk knowledge, looking for useful concepts and approaches to address critical issues of risk assessment and management. Neglecting risks leads the entrepreneurship to a state of myopia that makes it unable to predict or otherwise mitigate critical situations, increasing the vulnerability of all organization, constantly exposed to uncertainty and possible crisis.

The information collection and the learning process, increasing knowledge about nature and behavior of a particular event, can help the ability to manage risks and can play a key role in raising resources, tools or new knowledge which, without allow a precise prediction, will be able at least to reduce risk impact. 
However, the knowledge related to risk management, if misinterpreted, could lead to controversial situations. The larger uncertainty perception is, the stronger will be the people's inclination to not act; in other words, manager who is facing unknown situation usually tends to be more cautious, ready to come back or change his strategy just perceive discrepancy with respect of his expectations. At opposite, when the decision maker believes to know the event distribution, his strategic behavior is determined by a cognitive model built on its previous experience and thus makes him less sensitive to environment changes and to perception of every signal which could prevent or handle unexpected situations (extreme events) and crisis.

\section{Crisis Management and Vulnerability of the Organization}

We need a more stable and comprehensive concept of crisis in organizations facing complexity and uncertainty as well as a way to reduce risk by better prevention [21].

Crisis management and vulnerability of the organization are two very important concepts that have had considerable attention in management literature as the basis for defining processes of contingency planning such as operational crisis and crisis of legitimation for disaster recovery (bankruptcy, market breakthrough, change in leadership, fraud, etc.). Rarely has it focused on the processes by which the crisis has been generated, its longterm phases and embedded sources [22]. We need to develop a better perspective to explore the generation and nature of crisis events in organizations where, according to Smith [22] "management should not be seen as operating in isolation from the generation of those crisis that they subsequently have to manage, but rather as an integral component of the generation of such events". But we will see that the customer or the user becomes part of the strategic process to prevent and respond to crisis as "partners" of management and shareholders, sharing collective knowledge and information about value creation.

The main factors to create the pre-conditions for crisis and vulnerability of organizations are their interaction with the market and the often huge differentiation of this one; the growing importance of consumer expectations and perceptions which are closely connected with the image and reputation of the company and of the management; the technological innovations and long (or short) wave of change that pushes companies to change rapidly not only their products or markets, but also dealers, managers and organization; the nature of leadership, its stability and evolution, as in the case of a jump in family control or a huge change of shareholders [23].
For those reasons and many others that serve to create a complex portfolio of potential crisis scenarios, is necessary to outline the nature of the crisis management process and to know how the crisis evolve.

In the literature we can identify a focus on the development of contingency plans to cope with a range of crisis scenarios in terms of response teams, strategies for continuity service provision, and procedures to protect organizational assets and damage limitation. These are activities very important in stopping dangerous consequences of a crisis, but not useful to avoid it, as in the case of an organization's reputational harm, often irreversible. Effective crisis management should include a diffuse and systematic attempt to prevent crisis by occurring [22,24,25].

According to many authors, the notion of crisis starts from a circularity and interaction between different process stages not always linearly connected: crisis management, operational crisis, crisis of legitimation, process of organizational learning. In that view, a good preventative measure is creating resilience [21,25,26,27, 28]. This can be achieved within the organization by:

- trying to eradicate error traps as a path to explorative learning;

- developing a culture that encourages near-miss reporting;

- dealing with the aftermath of crisis;

- learning lessons from the event;

- defining an accountability to connect stakeholders for crisis potential [26,27].

According to Smith [22] we need a shift in the way of representing organizations' dynamic change and processes of management (before, during and after crisis) as nonlinear connections in space and time. This would allow us to explore pathways of vulnerability and erosion of defences (Figure 1):

1) Crisis of management: the problems are well known; managers believe that their organizations are safe, secure and well run because their short-term perspective sees the comparison between the cost of prevention and perceived costs of limitation and recovery derived from an underestimation of the second in respect to the first. The long-term view for many managers is rare, and often "hostages" of operational phases and controls are bypassed.

2) Operational crisis: many characteristics of the crisis are here often visible, but in many cases, contingent and temporarily defined. But we face often situations of deep incubation or latent conditions of crisis factors as if the organization is living in a permanent present, without a past and without a future. 


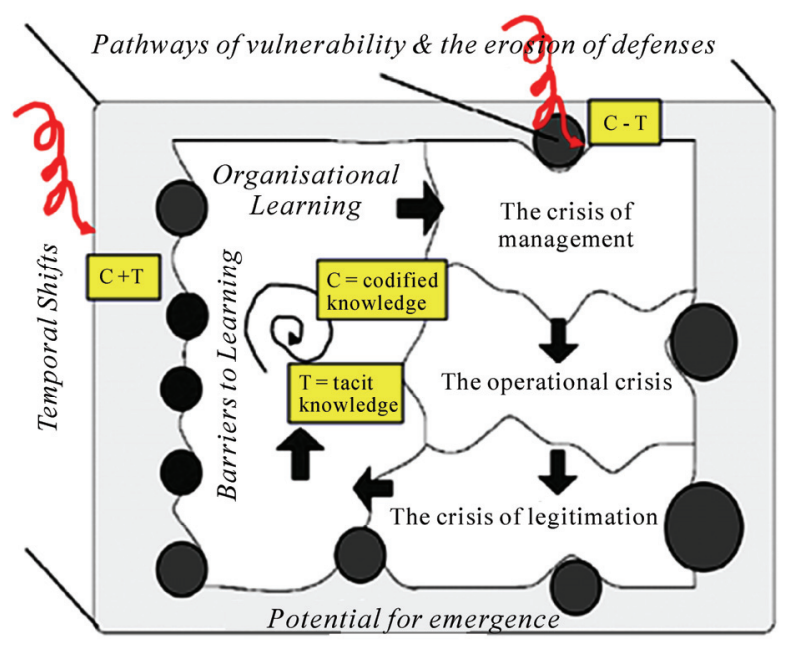

Figure 1. The nature of the crisis management process and the potential for emergence [22].

3) Crisis of legitimation: it starts internally but with immediate external effect attracting media coverage, governmental intervention or public inquiry as in the case of the recent bank crisis in Europe or great companies in USA. It could start from a failure in a specific product or service that causes a loss of customer confidence (and/or shareholders as well). At the same time, it will lead to erosion of the demand and probably to financial instability in the long run, with an impact on stability of shareholders and larger stakeholders too. The organizational learning will show a probable great fragility.

4) Processes of organizational learning: a crisis of legitimation leads to a crisis of procedure and practices and then to the lack of confidence of the middle management.

5) Crisis of interchange between tacit and codified knowledge: we can underline the role of knowledge diffusion as a factor of crisis in case of overestimation of codified knowledge with respect to tacit ones, considered as residual errors to remove by a hierarchical control mechanism. Interdependences between tacit and codified knowledge (or between voluntary and involuntary behavior) are the link with the past and future of the company. They represent both the main source of strategic competences in connection with its identity, and the architecture of organizational learning able to sustain more generations of entrepreneurs and managers as well as customers. This is key to describing one of the pathways of vulnerability.

Critical situation can be due to a lot of internal and external factors. Bad managed critical situation can turn into organizational crisis. Every risk, extreme event and uncertain situation, which cannot be forecasted, mitigated and controlled, is a potential factor of vulnerability and crisis for organization. It's important to exploit every innovation and tool which can make management process easier, more reliable and more responsible.

\section{Certainty, Uncertainty and Risk in Decision Making}

In economic context there are measurable uncertainty and unmeasurable uncertainty. If nothing you can say about uncertain event, risk may be thought as a measure- able uncertainty. Catastrophic event (rare event) is intermediate situation, it's not a fully unmeasurable uncertainty but an accurate foreseeing could be hard.

Extreme event is located in tails of distribution, it's featured by low probability of occurrence and high negative impact. No information on past behavior allow to exactly understand its dynamic evolution and consequently risk managers are unable to forecast it.

However mathematical models or statistical tools are not so useless as a lot of authors believe, if you judge they allow increasing knowledge accumulation. Better is your knowledge about a phenomena, better you can face it. According to Epstein [29] you can say a priori whether a risk is measurable or unmeasurable, it depends on the knowledge and information you have about it. For example, we are not yet able to predict an earthquake but we have learned to distinguish the potentially seismic zones according to their geological composition, to build using materials and techniques which sure high seismic resistance. Thus was possible on base of statistical analyses which allowed to verify where earthquakes happened with larger probability rate and to value the capacity of some materials to respond efficiently to stress actions. In other words, even if you cannot predict a catastrophic event, you can study its features and improve organization capability to adequately face it and to act fast and efficiently to reduce its impact when the event occurs.

In front to these situations, manager can choice different strategies. He may decide on responsible management of every risk or only more ordinary risks accounting. Many decision makers tend to overlook highly improbable events, though very dangerous; most of them usually think coverage costs related to the extreme event prediction are in the time higher than costs needed to face their occurrence. Probability estimation for an outcome based on judgment and experience may result successful but it depends on entrepreneurship or management capability and competence and in uncertain case on randomness. Mathematicals and statisticals allow to get an objective and more adequate probability estimation. Use of statistical techniques for objective management of risks effects on organizational culture and enhance pre- 
dictive capacity at every firm's level.

However, neglect to study extreme event distribution slows knowledge learning process and reduces organization' absorptive and adaptation capability.

Then overlooking behavior attends a high risk propensity of management and thus reflects negatively on the corporate culture, reducing liability and risk awareness and exposing the company to a higher probability of harmful events occurrence in the long time.

Knight [30] claimed "there is no difference for conduct between a measurable risk and unmeasurable uncertainty". Risk management doesn't mean necessarily to consider every potential risk, it regards awareness concept. Prudent management suggests you can choose to apply corrective measures and mitigation actions or rather decide consciously to neglect any specific risks. What is important a manager should make decisions within a rational and responsible approach based on probability of occurrence, predictability and impact estimation.

Then, a prudent and responsible decision maker should consider, in its evaluation and selection of strategies to follow, each time the reference context, differentiating his behavior from situations of certainty, risk or uncertainty. We talk about decision making under certainty when you can be sure, without doubt the authenticity of a case; in risk conditions, when you cannot be sure of the authenticity of a case but this one can be estimated with a certain probability rate; in uncertainty, when you cannot assess the authenticity of a case and cannot understand the probability that this is true, because you haven't information to make a reasonable estimate [31].

It is essential to distinguish between conditions of certainty, uncertainty or risk, because it has power over the chance to estimate probability of occurrence of an event and to determine the most appropriate and effective decision-making strategies in a specific situation. Under conditions of certainty, you will choose the action whose outcome provides the greatest usefulness; in a risky situation, you will value the greatest expected utility; in uncertain case, no decision can be considered completely reliable or reasonable and effects should be considered random [32]. In situation of uncertainty, manager doesn't know historical data suitability and future probability of occurrence, and there are infinite factors which could

\footnotetext{
${ }^{2}$ The term "generator" refers to the type of distribution that best estimate a well-defined set of data.

${ }^{3}$ See $[30,33]$. The distinction between risk and uncertainty is roughly that risk refers to situations where the perceived likelihoods of events of interest can be represented by probabilities measured by statistical tools using historical data, whereas uncertainty refers to situations where the information available to the decision-maker is too imprecise to be summarized by a probability measure $[30,33,34]$.
}

influence events evolution and change preliminary decision-making conditions.

Normally, in order to know the risks we are exposed, we need to choose the probability distribution form so well as to calculate the risks and find out the probability that a past event comes up again in the future. If you need a probability distribution to understand the future behavior, it's also true past events knowledge is necessary to determine the probability distribution. In other words, we are in a vicious circle [35].

One of the main problems is that risk management science has been addressed, in the last century, by an econometric and mathematical point of view, looking for potential models which were able to forecast loss distribution. What it's not clear is that risks of using a wrong probability distribution aren't obviously predictable and can be even more dangerous than those detected by chosen distribution tails [36].

The main problem of risk management is determined by the fact that general properties of the generators (distribution shape) $)^{2}$ usually outline a uncertain situation and not hazardous one. The worst mistake that can make the risk manager is to confuse uncertainty with risk, failing to define class and generator parameters. ${ }^{3}$

A generator has specific parameters that determine certain values of the distribution, allows analysts to calculate the probability that a certain event occurred. Usually the generator isn't known and there is no independent way to determine the parameters, unless you try to deduce from the past behavior of the generator. To estimate the parameters from historical data is still necessary to assume the generator class (Normal, Poisson, Binomial and other).

These estimates are more accurate as larger is the amount of data available. Logically, an inappropriate choice of the type of generator immediately affect the reliability of results (in terms of imprecise probability of occurrence of risky events).

The risk manager can run into several situations:

- the type of generator is easily identifiable and there is an adequate supply of past data;

- the generator can be considered reliable, but the lack of historical data makes impossible to correctly determine the moments;

- you cannot determine either the parameters or the general class of membership.

The last point is usually the dominant situation in which the relationship between expected risk and actual risk is still undetermined or accidental. There is a state of uncertainty, where it cannot be sure what will happen, or make any estimates on the probability of hypothetical 
cases.

Therefore, risk managers should not confuse uncertainty with risk, failing to define both the class generator and its parameters. A fact is making decisions under risk, where you know the distribution of a given phenomenon and the probability that a harmful event, another fact is to do it under conditions of uncertainty where the distribution is only conceivable and the probability of events is not accurately defined.

Further perspective is provided by recalling the role of differentiability in decision theory under risk, where utility functions are defined on cumulative distribution functions. Much as calculus is a powerful tool, Machina [37] has shown that differential methods are useful in decision theory under risk. Epstein [29] adds to the demonstration in Machina that differential techniques are useful also for analysis of decision-making under uncertainty.

Operational losses are usually forecasted using parametric and actuarial approaches as LDA (Loss Distribution Approach) or more cautious EVT (Extreme Value Theory) [38]. The main problems of this approaches concern the choice to reduce any event distribution to consolidated generator (normal, lognormal, GPD or other) and the need for consistent time series to obtain values appropriate risk.

The key attraction of EVT is that it offers a set of ready-made approaches to a challenging problem of quantitative operational risk analysis and try to make risks, which are both extreme and rare, appropriately modeled. Applying classical EVT to operational loss data however raises some difficult issues. The obstacles are not really due to a technical justification of EVT, but more to the nature of the data. EVT is a natural set of statistical techniques for estimating high quantiles of a loss distribution, which well works with sufficient accuracy only when the data satisfy specific conditions [39].

The innovation introduced by fractal model is the flexibility to adapt event distribution to real one without setting up the best generator. So you don't need to assume the shape of the generator because ifs allows to reproduce the structure of real distribution on different scales, exploiting the properties of self-similarity ${ }^{4}$ of fractals.

The fractal building is based on an innovative algorithm which is iterated a theoretically infinite number of times so that, in each iteration, the approximated distribution better estimates the real one. The IFS (Iterated Functions System) technique finds out the more appro-

\footnotetext{
${ }^{4}$ The basis of self-similarity is a particular geometric transformation called dilation that allows you to enlarge or reduce a figure leaving unchanged the form.
}

priate generator without a known model application [40]. Moreover the properties of fractals allow to estimate the event distribution in a reliable manner even if we have a lack of historical data.

Therefore the advantage of this approach is to give risk managers a tool to avoid the mistake of neglecting risks, regardless having to fix a suitable standard generator. A best estimate of an event risk level enhances the efficiency of its management, monitoring and control and reduces exposure of the organization.

\section{Sample and Methodology}

Our analysis is based on a two year operational loss data collection by an Italian banking group ${ }^{5}$. The dataset contains operational losses broken down by eight business lines and seven event types in accordance with the Basel II rules. The business lines are: Corporate Finance, Trading \& Sales, Retail Banking, Commercial Banking, Payment \& Settlement, Agency Services, Asset Management and Retail Brokerage. The event types are: Internal Fraud, External Fraud, Workplace Safety, Business Practice, Damage to Physical Assets, Systems Failures, Execution \& Process Management.

The available data consist of a collection of operational losses of an Italian banking group for a time period of two years divided into company code, type of business line, risk drivers, event type, amount of loss, date of occurrence, frequency of occurrence.

To have a significant analysis, we had to use a time horizon of one month instead of one year for our estimations (then, we have 24 observations of the aggregate monthly loss), and to focus only on the business line (we do not care of the event type); we analyze two different business lines: the traditionally $\mathrm{HFLI}^{6}$ retail banking [15] and the traditionally $\mathrm{LFHI}^{7}$ retail brokerage [41].

In Table 1 we show the descriptive statistics of retail banking and retail brokerage. In the retail banking business line we have 940 loss observations (high frequency business lines), the minimum loss is 430 euros, the maximum loss is 1066685 euros and the average loss is 13745 euros. In the retail brokerage business line we have 110 loss observations (low frequency business line) with a minimum loss of about 510 euros, maximum loss of 700000 euros and an average loss of 28918 euros (higher than retail banking business line).

We have used for our analysis three types of approaches: the traditional Loss distribution approach (LDA), the refined Extreme Value Theory (EVT) and the innovative fractal based approach Iterated function sys-

\footnotetext{
${ }^{5}$ For reasons of confidentiality we cannot use the group name

${ }^{6}$ High Frequency Low Impact

${ }^{7}$ Low Frequency High Impact
} 
Table 1. Descriptive statistics of retail banking (left) and retail brokerage (right).

\begin{tabular}{ccc}
\hline & $\begin{array}{c}\text { Retail Banking } \\
(940 \text { obs. })\end{array}$ & $\begin{array}{c}\text { Retail Brokerage } \\
(110 \text { obs. })\end{array}$ \\
\hline Min. & 438 & 509.6 \\
$1^{\text {st }}$ Qu. & 1302 & 1071.4 \\
Median & 3000 & 2124.3 \\
Mean & 13745 & 28918.3 \\
$3^{\text {rd }}$ Qu. & 8763 & 13375.0 \\
Max. & 1066685 & 700000.0 \\
\hline
\end{tabular}

tems (IFS).

First of all, we consider the actuarial-based Loss Distribution Approach. The goal of the LDA methodology consists in identifying the loss severity and frequency distributions and then calculate the aggregate loss distribution through a convolution between severity and frequency.

LDA is built upon two different distributions, estimated for every cell of the Business Line-Event Type1 matrix (ij): the distribution $F X_{i j}(x)$ of the random variable $X_{i j}$ which represent the loss amount trigged by a single loss event. This distribution is called loss severity distribution.

The distribution $P_{i j}(n)$ of the counting random variable $N_{i j}$, which probability function is $p_{i j}(k)=P(N=k) . P_{i j}$ is said loss frequency distribution and corresponds to:

$$
P_{i, j}(n)=\sum_{k=0}^{n} P_{i, j}
$$

These two distributions (which have to be independent from each other) represent the core o the LDA approach, and are used to obtain the operational loss calculated on (mainly) a one-year horizon (in our case one-month horizon) for the $i j$ cell:

$$
L_{i, j}=\sum_{n=0}^{N_{i j}} X_{i, j, n}
$$

The main approach to study extreme events is the Extreme Value Theory (EVT), which is a statistical methodology that allows analysts to handle separately the tail and the body of the distribution, so that the influence of the normal losses on the estimation of the particularly high quantiles can be eliminated. This technique was developed to analyze the behavior of rare events and to prevent natural catastrophes (i.e., floods or losses due to fires). There are two classes of distribution in particular which prove to be useful for modeling extreme risks: the first one is the Generalized Extreme Value (GEV) and the second one is the Generalized Pareto Distribution (GPD).

The Iterated Function Systems (IFS) represent a mathematically complex class of innovative and nonparametric fractal methods to create and generate fractal objects as an approach to shift between time towards space [40]. The fractals are geometric figures that can be represented at different dimensional levels, but they consist in an infinite replica of the same pattern on a smaller and smaller scale and so they are made up of multiple copies of themselves. This fundamental property of invariance is called self-similarity, one of the two principal properties that describe a fractal. The second property, not less important, is the indefiniteness, which is the possibility to fractionate virtually till infinite every part before going to the next one. Hence, to "draw" a fractal through a processor, the maximum number of iterations must be specified, because a finite time is insufficient to calculate a point of the fractal at infinite iterations.

One of the IFS possible fields of application is risk management, in which fractal methods are used as non-parametric estimation methods as an alternative to the Loss Distribution Approach (LDA) and the EVT [39]. These innovative methods can both interpret the data of loss in a more accurate way, and estimate and recreate the possible population from which they could derive, especially when there are few observations or data. The advantage of this approach is that the elaboration processes can be reduced while improving, at the same time, the capacity of estimation of the patrimonial requirements necessary to cover the expected and unexpected operational losses $^{8}$. IFS methods eliminate the necessity to analyze the distribution of frequency ${ }^{9}$, of severity ${ }^{10}$ and of the correlated convolutions between them, simulating directly the cumulative distribution of the aggregate losses and then applying the most efficient measure of risk $^{11}$. Consequently, they become a fundamental element in the analysis of losses, especially in correlation with the so called extreme losses, which are the losses that, due to their very low probability of occurrence, tend to be ignored, although their occurrence could determine catastrophic consequences.

In the next section, the fractal approach will be applied

\footnotetext{
${ }^{8}$ The expected loss is defined as the mean of the losses observed in the previous periods. The unexpected loss is defined as the difference between the VaR and the expected loss and, resulting more difficult to be represented in a model, represent for the business management an element of uncertainty which can be minimized only through ad adequate estimation.

${ }^{9}$ For distribution of frequency we intend the frequency of the occurrence of the events.

${ }^{10}$ For distribution of severity we intend the financial impacts generated by the losses.

${ }^{11}$ In our case Value at Risk
} 
to a specific case with the intent to demonstrate, at a practical level, what kind of information can emerge from the use of this approach and how this information can be used to improve the business management.

\section{Estimation by IFS Approach}

The non-parametric estimation we offered as an alternative way to LDA methodology and the Extreme Value Theory is the IFS fractal based approach which should be able to interpret loss data in the best possible way and to simulate a population (see Figure 2) from which our observations could come and especially in the presence of a few observations and missing data (as in the case of retail brokerage). The advantage of this approach is to eliminate the analysis on frequency and severity distribution with related convolution and the previous choice of a known distribution to directly simulate the cumulative distribution function of the aggregate operational losses and then apply a risk measure to calculate capital requirements to cover operational losses in the business lines we studied.

The Iterated Function Systems, designed originally from Barnsley for the digital image transfer [42], depth by Forte and Vrscay [43] for solving inverse problems, and used by Iacus and La Torre for their estimation and simulation mathematical property of probability functions [40] and, finally, adapted to the capital requirements calculation of operational risk, have shown over other methods (such as LDA and EVT) more relative efficiency in terms of ability to reconstruct a population of losses.

We used the function arctang for transforming the values of loss in values between 0 and 1 to allow the IFS approach, that functions on a finite support (in our case precisely between 0 and 1), to estimate the operational cumulative distribution function starting from the empirical distribution function (EDF).

First, we have to demonstrate that IFS approach is better than LDA and EVT to reconstruct a population from

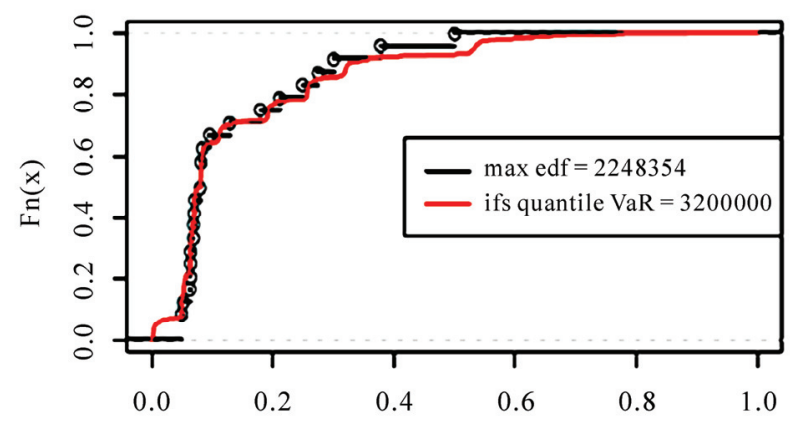

Figure 2. Example of IFS simulation in retail banking. which our information could come and especially in the presence of few data. The methodology to demonstrate the capability of the IFS estimator to reconstruct the original population is to calculate a statistical distance from the original distribution of the IFS simulation and comparing the results obtained with the classic method used in the actuarial science (LDA) and the most innovative method of Extreme Value Theory. For our analysis we use observations of operational losses caused by fires. The database Danish Fire Losses in literature has been used to test both classical techniques such as LDA that EVT as the newest techniques and lends itself very well to the study of extreme and complex events as demonstrated by McNeil et al. [44] and therefore it represents a good test for analyzing and evaluating the relative efficiency of the fractal approach versus parametric techniques such as LDA and EVT. This database contains daily observations of loss arising fire in Denmark in millions of Danish crowns from January 1980 to 1990 and is free downloadable in the package QRMLib by $\mathrm{R} 2.10 .0$ project. We proceed extracting random samples of different sizes from the database and then simulate the real distribution of losses through the use of IFS maps for small sample size $(\mathrm{n}=10,20,30)$ and medium sample (n $=50,100,250)$. Finally we compare the results obtained by IFS approach in terms of $\mathrm{AMSE}^{12}$ distance with the results obtained by LDA and EVT approaches.

In Table 2 we show the behavior of the AMSE index ${ }^{13}$ (calculated over 100 simulations for each sample size).

The indices show us how IFS approach is more efficient for small sample size than LDA and EVT, while for samples of medium and large size we find an asymptotic behavior between LDA approach and IFS one.

Secondly, we apply this new fractal methodology for estimating enterprise capital requirements that a firm must allocate to cover operational risks. We know the main problem for the operational risk analysis is the lack of information and the lack of data, so we use the power and the capability of the fractal objects to reconstruct a population starting from this lack of information to better understand the real risk profile of the firm and to improve the decision making process inside the company. In Table 3 we show the result of non-parametric estimation made by IFS approach for the retail banking and retail brokerage business lines compared with the value obtained by LDA VaR, EVT VaR and the maximum value of the empirical distribution function.

In the two business lines in which we were able to apply all the three approaches proposed in this paper, we

\footnotetext{
${ }^{12}$ Average mean square error

${ }^{13}$ Lower is the value of the AMSE index better is IFS approach versus LDA and EVT
} 
Table 2. Relative efficiency of IFS estimator over LDA and EVT.

\begin{tabular}{ccc}
\hline$n$ & AMSE over LDA & AMSE over EVT \\
\hline 10 & 65.13 & 24.05 \\
20 & 73.52 & 31.26 \\
30 & 75.15 & 25.95 \\
50 & 79.33 & 22.27 \\
100 & 111.12 & 21.05 \\
250 & 115.67 & 21.11 \\
\hline
\end{tabular}

Table 3. Value at Risk by different approaches.

\begin{tabular}{ccccc}
\hline $\begin{array}{c}\text { Business } \\
\text { line }\end{array}$ & LDA VaR & EVT VaR & $\begin{array}{c}\text { IFS } \\
\text { VaR }\end{array}$ & Max EDF \\
\hline $\begin{array}{c}\text { Retail } \\
\text { banking } \\
\begin{array}{c}\text { Retail } \\
\text { brokerage }\end{array}\end{array}$ & 1531369 & 4420998 & 3200000 & 2248354 \\
\hline
\end{tabular}

obtained, with IFS method, estimates in the middle between the Extreme Value Theory which tends to overestimate widely the capital requirements and the Loss Distribution Approach which tends to underestimate the requirements (for example in the case of retail banking). Another IFS advantage is the ease of use, meaning that you do not need to simulate a distribution for the severity and for the frequency and the related convolution, but is enough to measure directly the aggregated operational loss distribution (monthly in our case given the number of observations available) and apply to this distribution a measure of risk.

The strength of this fractal approach has demonstrated its ability to capture the true risk profile of a company even with lack of data.

Of course not everything is positive, but we must take into account and know perfectly well there are inherent disadvantages and limitations that make each model less accurate and sensitive to errors. Firstly like all nonparametric approaches, the IFS are very sensitive to the sample data used. Several samples extracted from the same population can lead to estimates and simulations widely different between them. It is a good idea to use a very large number of simulations in order to obtain consistent estimates.

\section{Conclusions}

In recent years, there has been increasing concern among researchers, practitioners, and regulators over how to evaluate models of operational risk.

The actuarial methods (LDA) and the Extreme Value
Theory (EVT) constitute a basis of great value to fully understand the nature and the mathematical-statistical properties of the process underlying the operational losses, as regards the severity of losses, the frequency of losses and the relationship between them.

Several authors have commented that only by having thousands of observations can interval forecasts be assessed and traditional techniques need a large amount of data to be precise and effective. However, currently available data are still limited and traditional methodologies fail to grasp the correct risk profile of firms and financial institutions, as shown the empirical analysis undertaken in this article. With IFS technique you can estimate appropriate risk value even if you have just few data. Increasing data availability, IFS outcomes tend to be as suitable as traditional methods.

So, we can say that the study, the analysis and the improvement of innovative methods such as Iterated function systems (IFS) is a valuable support for measurement and management the operational risk alongside actuarial parametric techniques. Not only that, their use is not limited only in the next years, pending more complete series. Their use should include those areas of operational risk, which is by definition heterogeneous and complex, have a limited numbers of events, but their impacts can, however, be devastating for firms and stakeholders. Precisely for this reason the use and improvement of these innovative tools must continue for the foreseeable future because there will always be the need in this area of innovative approaches able to predict, with good approximation, situations starting from this lack of data and reconstruct a precise and faithful population to integrate and assess the results of other different approaches.

As such as every mathematical method, IFS could help risk management making quicker knowledge learning process but what is really important is a careful and prudent management behavior.

Thus we can conclude, firstly, mathematical approaches are effective only when they are integrated and shared in a responsible corporate culture. Secondly, IFS as a nonparametric method is sensitive to the composition of used sample; for an appropriate estimation of operational loss with IFS you should reapply the methods more times and then estimate an adequate average level. More cautiously you apply mathematical techniques, more probable is a accurate estimation of hedging value of the total operational risk. Finally, being the first time IFS is applied to operational risk, future improvements are probable.

In our intentions is we would extend the application of this methods to financial and credit risk. However this goal needs we are able to use IFS to estimate not just probability function but density one. The main problem it 
requires to apply a Fast Fourier Transform, as shown by Iacus and La Torre [40].

\section{REFERENCES}

[1] D. Foray, "Economics of Knowledge," MIT Press, 2004.

[2] S. Vicari, "Conoscenza e impresa," Sinergie rivista di studi e ricerche, Vol. 76, 2008, pp. 43-66.

[3] D. J. Teece, "Managing Intellectual Capital," Oxford University Press, Oxford, 2000.

[4] E. Altman, "Financial Ratios, Discriminant Analysis and the Prediction of Corporate Bankruptcy," Journal of Finance, Vol. 23, No. 4, 1968, pp. 569-609.

[5] L. Pilotti, "Le Strategie dell'Impresa," Carocci, Roma, 2005.

[6] R. M. Grant, "Toward a Knowledge-Based Theory of the Firm," Strategic Management Journal, Vol. 17, No. Special Issue, 1996, pp. 109-122.

[7] J. A. Schumpeter, "Business Cycles," McGraw-Hill Book Company, New York, 1939.

[8] A. Pagani, "Il nuovo imprenditore," Franco Angeli, Milano, 1967.

[9] M. Haller, "New Dimension of Risk: Consequences Management," The Geneva Papers on Risk and Insurance, Vol. 3, 1978, pp. 3-15.

[10] G. Dickinson, "Enterprise Risk Management: Its Origin and Conceptual Foundation," The Geneva Papers on Risk and Insurance, Vol. 26, No. 3, 2001.

[11] E. Rullani, "Economia del rischio e seconda modernità," In S. Maso, Ed., Il rischio e l'anima dell'Occidente, Cafoscarina, Venezia, 2005, pp. 183-202.

[12] G. M. Mantovani, "Rischio e valore dell'impresa. L'approccio contingent claim della finanza aziendale," Egea, Milano, 1998.

[13] G. M. Golinetti, L. Proietti and G. Vagnani, "L'azione di governo tra competitività e consonanza," In G. M. Golinelli, Ed., Verso la scientificazione dell'azione di governo. L'approccio sistemico al governo dell'impresa, Cedam, Padova, 2008.

[14] L. Proietti, "Rischio e conoscenza nel governo dell'impresa," Sinergie rivista di studi e ricerche, Vol. 76, 2008, pp. 191-215.

[15] BIS, "International Convergence of Capital Measurement and Capital Standards," Basel Committee on Banking Supervision, 2004.

[16] P. Davidson, "Money and the Real World," The Economic Journal, Vol. 82, No. 325, 1972, pp. 101-115.

[17] P. Streeten, "The Cheerful Pessimist: Gunnar Myrdal the Dissenter (1898-1987)," World Development, Vol. 26, No. 3, 1998, pp. 539-550.

[18] K. J. Arrow, "Alternative Approaches to the Theory of Choice in Risk-Taking Situations," Econometria, Vol. 19, 1951, pp. 404-437.

[19] H. M. Markowitz, "Foundation of Portfolio Theory," Journal of Finance, Vol. 46, No. 2, 1991, pp. 469-477.
[20] U. Beck, "Risk Society: Towards a New Modernity," Sage, London, 1992.

[21] D. Elliott and D. Smith, "Football Stadia Disaster in the UK: Learning from Tragedy," Industrial and Environmental Crisis Quarterly, Vol. 7, No. 3, 1993, pp. 205-229.

[22] D. Smith, "Business (not) as Usual: Crisis Management, Service Recovery and the Vulnerability of Organizations," Journal of Services Marketing, Vol. 19, No. 5, 2005, pp. 309-320.

[23] P. M. Allen and M. Strathern, "Evolution, Emergence and Learning in Complex systems," Emergence, Vol. 5, No. 4, 2003, pp. 8-33.

[24] D. Smith, "Beyond Contingency Planning: Towards a Model of Crisis Management," Industrial Crisis Quarterly, Vol. 4, No. 4, 1990, pp. 263-275.

[25] D. Smith, "On a Wing and a Prayer? Exploring the Human Components of Technological Failure," Systems Research and Behavioral Science, Vol. 17, No. 6, 2000, pp. 543-559.

[26] J. T. Reason, "The Contribution of Latent Human Failures to the Breakdown of Complex Systems," Philosophical Transactions of the Royal Society of London, Series B, Vol. 327, No. 1241, 1990, pp. 475-484.

[27] J. T. Reason, "Managing the Risks of Organizational Accident," Ashgate, Aldershot, 1997.

[28] C. Sipika and D. Smith, "From Disaster to Crisis: The Failed Turnaround of Pan American Airlines," Journal Contingencies and Crisis Management, Vol. 1, No. 3, 1993, pp. 138-151.

[29] L. G. Epstein, "A Definition of Uncertainty Aversion," Review of Economic Studies, Vol. 66, No. 3, 1999.

[30] F. Knight, "Risk, Uncertainty and Profit," Houghton Mifflin, Boston and New York, 1921.

[31] D. Pace, "Economia del rischio. Antologia di scritti su rischio e decisione economica," Giuffrè, 2004.

[32] J. V. Neumann and O. Morgenstern, "Theory of Games and Economic Behavior," Princeton University Press, 1944.

[33] J. M. Keynes, "The General Theory of Employment, Interest and Money," Macmillan, New York, 1936.

[34] J. M. Keynes, "A Treatise on Probability," Macmillan, New York, 1921.

[35] N. N. Taleb and A. Pilpel, "On the Unfortunate Problem of the Nonobservability of the Probability Distribution," 2004. http://www.fooledbyrandomness.com/knowledge.pdf

[36] R. Lowenstein, "When Genius Failed: The Rise and Fall of Long-Term Capital Management," Random House, New York, 2000.

[37] M. J. Machina and D. Schmeidler, "A More Robust Definition of Subjective Probability," Econometrica, Vol. 60, No. 4, 1992, pp. 745-780.

[38] J. Neslehová, P. Embrechts and V. Chavez-Demoulin, "Infinite-Mean Models and the LDA for Operational Risk," Journal of Operational Risk, Vol. 1, No. 1, 2006, pp. 3-25. 
[39] P. Embrechts and G. Samorodnitsky, "Ruin Problem and How Fast Stochastic Processes Mix," The Annals of Applied Probability, Vol. 13, No. 1, 2003, pp. 1-36.

[40] S. Iacus and D. L. Torre, "Approximating Distribution Functions by Iterated Function Systems," Journal of Applied Mathematics and Decision Sciences, Vol. 2005, No. 1, 2005, pp. 33-46.

[41] M. Moscadelli, "The Modeling of Operational Risk: Experience with the Analysis of the Data Collected by the Basel Committee," Temi di discussione, No. 517, 2004.
[42] M. Barnsley and L. Hurd, "Fractal Image Compression," AK Peters. Ltd, Wellesley, MA, 1993.

[43] B. Forte and E. Vrscay, "Inverse Problem Methods for Generalized Fractal Transforms," In: Y. Fisher, Ed., Fractal Image Encoding and Analysis, Springer-Verlag, Trondheim, 1995.

[44] A. J. McNeil, R. Frey and P. Embrechts, "Quantitative Risk Management: Concepts, Techniques, and Tools," Princeton University Press, Princeton, 2005. 